\title{
BUSCANDO RESPUESTAS AL RETO DE LA DIVERSIDAD DESDE LA PEDAGOGÍA CRÍTICA
}

\author{
M.Sc. Paulette Barberousse*
}

Repensar la diversidad, desde la óptica de la pedagogía crítica, consiituye uno de los principales desafios que se le presentan a la educación contemporánea dado que el tema de la alteridad se ha transformado en un imperativo que todos debemos asumir en tiempos y espacios de globalización y posmodernidad. La misma diversidad que se presenta en la sociedad, se reproduce, con sus especificidades, en la escuela. El problema es que en la sociedad la diversidad es considerada desigualdad y en la escuela como diversidad y ésta puede enmascarar y disfrazar "pedagógicamente" las desigualdades. Debemos reconstruir y repensar la escuela desde la diversidad para la igualdad bajo el mandato de compensar las desigualdades.
Rethinking diversity from the point of view of critical pelagogy constitutes one of the main challenges of contemporary education, given that we all must assume the topic of altering in times of globalization and post-modernism. The same diversity which is present in society, is also a specific factor at school. The problem is that diversity in society is considered as inequality and at school it means diversity, which can mask "pedagogical" inequalities. We must rebuild and rethink school from the diversity for equality under the mandate of compensating inequalities.

* Posee una Maestría en Ciencias Históricas y Filosóficas de la Universidad Babes-Bolyai de la República de Rumanía. Obtuvo también una Maestría en Ciencias de la Educación con énfasis en Educación de Adultos de la Universidad de Costa Rica. Por formación, su experiencia como docente, investigadora y productora se inscribe en el marco de las temáticas de fundamentación teórica de las ciencias pedagógicas (Filosofía Educativa, Epistemología Educativa, Teoría Educativa, Sociopedagogía, Historia y Sociología Educativa). 
Iis necesario aprender a navegar en un océano de incertidambres a través de archipiélages de certe:a.

Edgar Morin

El tema de la diversidad y la alteridad tiene tantas lecturas como lectores, y abordarlo implica, más que la búsqueda de una "solución", la promoción de reflexiones. Son más preguntas que respuestas, pero sin ellas el silencio se vuelve ensordecedor. Se necesita el debate integrador, donde las diferencias no impidan reconocer lo contrario: la uniformidad, la homogeneización, la segregación y la adaptación empobrecedora. La historia del ser humano está construida con base en la diferenciación, en la complejidad de reconocerse semejante y, al mismo tiempo, distinto al prójimo, en una dialéctica que incluye ex tremos como la xenofobia y el racismo. De más está el advertir lo bien que le vendría a la sociedad contemporánea un diálogo más tolerante con lo ajeno.

Repensar la diversidad es un imperativo que todos debemos asumir habida cuenta que el espacio democrático se conquista accediendo a la duda, a la oscilación de vivir permanentemente buscando una estabilidad siempre esquiva. Se trata, en última instancia, de oponer a la adaptación quejosa, una desadaptación creativa.

Cualquier dinámica de construcción identitaria lleva implícita la tensión pertenencia/no pertenencia. Todas las culturas procesan exclusiones, mejor dicho, poseen rasgos excluyentes. Dejarse sorprender por el otro, discernir que no tiene la obligación de parecerse a mí, demanda un largo trabajo psíquico, subjetivo y político. Más que un punto de partida es un punto de llegada. El ser humano nace socialmente inmaduro y su vínculo primario es con lo reconocible, lo similar. Todo debe resonar al unísono con él para ser aceptado y, lo que no encaja con ese patrón, es rechazado por estridente y amenazador.

La diversidad no solamente es un producto de la globalización, sino su otra cara inevitable. Con la relativización de la dimensión espacio-temporal provocada por la revolución tecnológica (instalando la aldea global, la virtualidad y el puro presente) las diversidades, lejos de morir, gozan de muy buena salud. Por suerte. Hablando sobre "la igualación en la desigualdad", Eduardo Galeano (1999) nos comenta:

...Este mundo del fin del siglo, que convida a todos al banquete pero cierra la puerta en las narices de la mayoría, es al mismo tiempo igualador y desigual. Nunca el mundo ha sido tan desigual en las oportunidades que brinda, pero tampoco nunca ha sido tan igualador en las ideas y costumbres que impone. La igualación obligatoria, que actúa contra la diversidad cultural del bicho humano, impone un totalitarismo simétrico al totalitarismo de la desigualdad de la economía, 
impuesto por el Banco Mundial, el Fondo Monetario Internacional y otros fundamentalistas de la libertad del dinero. En el mundo sin alma que se nos obliga a aceptar como único mundo posible, no hay pueblos sino mercados; no hay ciudadanos sino consumidores; no hay naciones sino empresas; no hay ciudades, sino aglomeraciones; no hay relaciones humanas, sino competencias mercantiles. (pág. 28)

Coincidamos en que la globalización conlleva la imposición de la homogenización y la supremacía de los modelos culturales, políticos y económicos de los países dominantes. Por otro lado, la identidad implica necesariamente el reconocimiento de la diversidad que debe preservarse en sus hábitats ecoculturales. Se trata de percibir la realidad desde una nueva mirada y desde una profunda reforma del pensamiento: en todos los ámbitos de la existencia social donde hay vida hay desequilibrio, variedad, inestabilidades, indeterminaciones e impredecibilidades que constituyen connotaciones positivas porque conforman los rasgos esenciales y constitutivos por antonomasia de la vida.

Percibimos al mundo desde un marco vital, desde nuestras realidades sociales y culturales que sólo son constructos históricos y culturales. Una de las principales representaciones colectivas que comparte la sociedad es, sin dudas, la escolarización que, como práctica institucionalizada, cumple el propósito de difundir el conocimiento y la cultura y construye un universo simbólico de significados, valores, deseos, aspiraciones y expectativas que conforman nuestra forma de sentir, pensar y actuar. Representa un fenómeno social de vital importancia para la construcción del estado moderno y se opone a las corrientes que intentan desacreditar su papel y lo disminuyen a la categoría de "mercancía" o "capital humano". El saber escolarizado no desarrolla las potencialidades, sino que genera las capacidades, sin olvidar que no toda enseñanza viene de afuera, el sujeto también aprende de sí mismo. Desde siempre, el optimismo pedagógico de fuerte raigambre en el pensamiento ilustrado y rousseauniano, que ayuda a conformar y viabilizar la idea de estado-nación, se transforma en un derecho universal que involucra a los sujetos en el mundo y en la vida.

Según Larrosa (1995) las prácticas educativas y sus dispositivos pedagógicos construyen y median las relaciones del sujeto consigo mismo y la experiencia escolar deja su impronta imborrable en el sujeto. Siempre hemos reconocido que la escuela promueve los procesos civilizatorios de individualización, de identificación y de socialización de los sujetos involucrados en su dinámica cultural. Este hecho constituye un fenómeno social que se traduce en procesos psíquicos y cognitivos de cambio, modificación y tensión creativa y constructiva que no deseamos uniformizar so pena de violentar la diversidad cultural de 
pueblos y sujetos. Como proyecto humanizador, la escuela debe aportar una mirada crítica acerca del mundo construido y sistematizar los saberes en función de una racionalidad abierta (no de una estéril racionalización) que propicie la apropiación del capital cultural externo en capital o competencia cultural para los sujetos y que los capacite para su inserción y para su singularización (incluyendo. al decir de Gimeno Sacristán. la autoexclusión o automarginación consciente).

La educación como "la fuerza del futuro" (UNESCO) constituye uno de los instrumentos más poderosos para generar el cambio y acompañar a nuestro pensiamiento de manera que enfrente la complejidad creciente, la rapidez de los cambios y lo imprevisible que caracterizan nuestro mundo. La necesidad de reforma del pensamiento humano la reafirma Edgar Morin(2000) cuando explica:

Existe cada vez más inadecuación y distancia entre nuestros saberes parcelados, separados y compartimentados (cada disciplina va por un lado) y los problemas y realidades que cada vez son más complejas, multidimensionales, pluridisciplinarias, transnacionales y planetarias. Esta situación hace que no veamos los problemas complejos, las interacciones y retroacciones entre las partes y el todo, las entidades multidimensionales y los problemas esenciales. (pág. 17)

De esta manera, el conocimiento resultante de una aproximación inter y la transdisciplinaria, genera pensamiento capaz de significar la realidad desde un conocimiento contextualizado que permite "alimentar un pensamiento que puede considerar la situación humana, en la vida, en la tierra y en el mundo y que puede afrontar los grandes desafíos de nuestro tiempo.. Se trata de armar cada mente en el combate vital para la lucidez.." (en negrito nuestro, 3)

No podemos permitirnos el lujo de marginar a nadie de la capacidad de significar su propia realidad como sujetos en proceso permanente de construirse como tales: nuestro currículo escolar, basado en la separación del conocimiento en disciplinas, agobiado por la excesiva escolarización y presencialidad, expropia al ser humano de su capacidad de lograr un conocimiento unificado, integral, holístico, e inhibe además el crecimiento y desarrollo de nuestros espacios interiores, únicos garantes, al fin de cuentas, de nuestra especificidad humana. Debemos aprehender la realidad y sus objetos en sus contextos, sus complejidades, sus conjuntos y mostrar " la unidad indisoluble entre la unidad y la diversidad en todo lo que es humano". Tal imposición nos obliga a detenernos a pensar en las consecuencias que este hecho conlleva para el discurso de la atención a la diversidad.

A través de los mecanismos de marginación, sometimiento e imposición de una única manera de pensar y de ver, el mundo se visualiza como homogéneo donde lo diferente y lo diverso no tiene cabida. 
En este sentido, el pedagogo español Jorge Rogero(2000) nos amplía:

...el tiempo en que vivimos se caracteriza como el más difícil de entender. Asistimos a un cambio de época y nos encontramos en plena crisis de inteligibilidad con una profunda falla entre la necesidad de comprender y las herramientas conceptuales necesarias para ello. A la desaparición de las certezas y de un proyecto colectivo se suma un mundo zarandeado por formidables mutaciones tecnológicas, por la persistencia de desórdenes económicos y por el aumento de los peligros, miedos y riesgos que reinventan todas las búsquedas de la seguridad que se necesita para poder seguir viviendo. (pág. 54)

Si el mundo es un teatro, nuestro escenario se caracteriza por la paradoja y la contradicción y son tiempos, según Follari, de "cambalache ideológico y travestismo político". Sus bambalinas y decorados: sociedad del conocimiento y la información versus desinformación y pensamiento único; globalización neoliberal versus organizaciones que luchan por la ciudadanía planetaria; estructuras supranacionales versus el redescubrimiento de nuevas identidades; fundamentalismos y movimientos religiosos de liberación; vaciamiento de la democracia versus el resurgimiento de la sociedad civil; tensiones internacionales y conflictos locales; defensa del espacio público versus afianzamiento del individualismo; crisis del trabajo y exclusión social; mayor creación de riqueza y pésima distribución, posmodernidad versus valores imperecederos; sociedad del vacío, de lo efímero, versus búsquedas del sentido; resurgir de una nueva ética y el crepúsculo del deber; la sociedad del hedonismo versus pérdida del sentido de lo colectivo; la sociedad de la imagen, de lo virtual, de lo irreal versus contacto y proximidad física; sociedad de la solidaridad con lo lejano versus la incomunicación y el desconocimiento del prójimo.

Esta sociedad económicamente dinámica y socialmente excluyente que realiza una piratería legalizada de los bienes comunes de la humanidad y reduce a la persona humana a "recurso humano", donde lo económico prevalece sobre lo político y lo político se subsume a lo económico, cuyo lema parece ser "mas mercado, menos estado"; esta sociedad neoconservadora y pragtmática, esta sociedad de la "calidad total" que promueve el "americanismo" como fase superior del capitalismo y modelo de vida y que practica la cultura de la meritocracia que califica de utópico todo intento de cambio y transformación, se ha trastocado en una sociedad del riesgo que propone la enseñanza sin educación.Rogers, J (2000)

J.M. Mardones(1998) en su libro "Desafíos para recrear la escuela" dice: 
...la sociedad ofrece zonas oscuras o puntos negros que son trampas para el individuo que transita por ellas...La tecnoeconomía, la industrialización, la burocracia de la administración del estado moderno, verdaderos motores de la modernización, han derivado en riesgos la misma vida que han ayudado a construir... hemos pasado de una sociedad de miedos identificables a una sociedad de riesgos insertos en el dinamismo de la propia sociedad ... el riesgo abraza la globalidad de la sociedad moderna ... el ser humano y la sociedad se han vuelto vulnerables ... inocula el miedo, la sensación de inseguridad .No es extraño que de nuevo, como defensa compulsiva, abunden las reacciones crispadas y violentas, reacciones defensivas frente al peligro que son los otros, lo desconocido, lo diverso, lo no familiar. (pág. 24)

Los avances del conocimiento científico no han generado mayor racionalidad en la vida diaria así como podemos afirmar que la escuela no transmite la actitud científica dado que introduce bruscamente el conocimiento científico sin contextualizarlo y relacionarlo con el conocimiento narrativo que implica sucesión y descripción de acontecimientos con carácter normativo.

En este sentido, Juan Delval (2000) apunta:

Por ello, en la actualidad, la escuela es una institución paradójica y contradictoria, pues mientras parece encaminarse a unos fines, en definitiva se orienta hacia otros. Se pretende que la escuela sea un instrumento para la igualación social, pero sabemos que mantiene las desigualdades y que el mayor fracaso escolar se produce entre los que vienen de los sectores mas desfavorecidos. Pretende preparar a los individuos para ser autónomos y ciudadanos libres en una sociedad democrática y de hecho promueve la sumisión y aceptación de la autoridad que tiene el poder. Pretende iniciar a los alumnos en la ciencia, y solo consigue que se repitan, sin entenderlos, algunos resultados. (pág. 36)

La educación siempre se ha ligado con la emancipación, con la liberación y emancipación humana. Lo que se debe abolir, pareciera susurrarnos el autor, no es la pobreza, sino la extrema riqueza.

Partamos de la base que la misma diversidad que se presenta en la sociedad se reproduce en la escuela. Por esta razón, en los últimos años, el tema de la diversidad se ha adueñado del debate pedagógico generando posturas disímiles en su comprensión y formas de actuar, no pocas veces antagónicas aún cuando 
blanden las mismas consignas. La misma desigualdad que existe en la sociedad se refleja, no mecánicamente sino guardando las especificidades del contexto pedagógico, en la escuela. El problema es que en la sociedad la diversidad es considerada como desigualdad y en la escuela como diversidad. Igualdad se opone a desigualdad, no a diferencia y diversidad. No "pedagogicemos" los problemas sociales ya que la diversidad disfraza, enmascara y profundiza las desigualdades. La diversidad es lo normal, lo habitual, es lo positivo y enriquecedor. Es consustancial al género humano y un objetivo socialmente deseable. Ello nos exige creatividad, coherencia, compromiso, implicación y esperanza. Sin embargo hemos considerado normal lo que no es: la desigualdad que domina en la vida, en la sociedad y en la escuela. La escuela pública puede actuar como equilibradora de los desequilibrios sociales provocados por la desigualdad y la marginación, es decir, trabajar la diversidad para superar la desigualdad entendiendo que la igualdad, soporte ético de la pedagogía de la diversidad, no significa uniformidad y bajo el mandato de compensar las desigualdades.

Al considerar a la escuela (en sentido genérico de institución escolar) reproductora del sistema social estamos evadiendo la posibilidad de transformarla en agente de cambio con relativa autonomía y especificidad. La educación debe ser percibida como espacio de resistencia: de condicionada se puede transformar en condicionante. Debemos de plano rechazar las posturas academicistas que tratan de "adaptarse" al statu quo, al igual que las posturas psicologizantes y didactistas que pretenden superar el fracaso escolar mediante el maquillaje de cambios exclusivamente metodológicos y que lo hacen recaer en el individuo cuando en realidad cumplen la misión de legitimar desigualdades sociales. Debemos reconstruir y repensar la escuela desde la diversidad para la igualdad.

En la praxis pedagógica lo diverso es lo habitual, lo excepcional es lo uniforme. Se torna necesario abordar el problema de la diversidad como un problema de desigualdad ante un currículo escolar que no es neutral ya que se asienta en su carácter y contenido ideológico. Esta ausencia de neutralidad le confiere a esta problemática una dimensión ideológica que afecta tanto las decisiones macropolíticas como a la intervención docente, guiada esta última por valores y creencias que conciben la homogeneidad como el paladín de la calidad educativa cuando, en realidad, la heterogeneidad, enmarcada en un currículo comprensivo, abierto y flexible, único y diverso que se adapte al alumno y no a la inversa, constituye la única garantía de una educación formativa y vivencial.

La atención a la diversidad entendida desde la pedagogía crítica como lucha por la igualdad y como lucha por la construcción de una educación integradora y compensadora de desigualdades, implica elaborar proyectos educativos que 
contribuyan a compensar y superar las desigualdades en lugar de reproducirlas o aumentarlas; construir comunidades de aprendizaje cooperativo y reflexivo donde el diálogo se erija como principal mecanismo de construcción de significados interactivos e intersubjetivos del conocimiento. Para cumplir con tal asignación son necesarios equipos de profesores comprometidos con la educación pública que sean capaces de revolucionar su pensamiento y, sobre todo, su sensibilidad pedagógica como herramientas básicas de los procesos de crecimiento, de hominización y humanización a fin de lograr la tan ansiada realización humana. Es indispensable que conjuguen sus esfuerzos con las familias, los agentes y organizaciones de la sociedad civil, con el contexto real y afectivo de todos los integrantes de la comunidad educativa en pro de una educación para vivir con dignidad.

Se trata de favorecer la igualdad de oportunidades y la comprensión de las desigualdades mediante una enseñanza comprensiva e inclusiva como garante de la atención a la diversidad en sus más variadas modalidades y expresiones: cultural, étnica, de género, cognitiva, social, económica, política, religiosa, de acceso a información, de lugar que se ocupa en el proceso productivo, ética, moral, de sensibilidad, afectiva y emocional. Sentimos distinto, necesitamos distinto, actuamos distinto, pero podemos estimarnos y querernos tal como somos si logramos establecer cauces de participación e interconexión entre las más variadas maneras de vivir, celebrar o mirar la vida.

Si la orientación y esfuerzos durante la década de los ochenta del debate pedagógico se centró en la búsqueda de una educación equitativa e igualitaria para todos y todas a través del aprendizaje significativo, la tendencia de los noventa pone acento en la adaptación a la diferencia y la diversidad para lograr la transformación del contexto mediante el aprendizaje dialógico que conforme agrupamientos flexibles e itinerarios formativos diversos que permitirían el nacimiento de una escuela plural que reconstruya críticamente la realidad y nuestra propia identidad desde una perspectiva liberadora con sentido personal y social, que forme para la participación democrática y ciudadana. Al decir de Brunner, la educación es una forma de vivir la cultura. No podemos, sin embargo, dejar de externar nuestra preocupación que el discurso sobre la atención a la diversidad se transforme hoy en un discurso que inmovilice, en la práctica, la potencialidad transformadora de la escuela al adormecer su dimensión liberadora, justificando su status quo dotándolo de una perspectiva explicativa y científica de las desigualdades sociales y culturales. Y lo que suponemos más peligroso aún: creer y convencernos que podemos solucionarlo desde y exclusivamente desde el ámbito pedagógico. 
En este sentido, comentando el salto que significa la orientación comunicativa a expensas de la constructivista, Ramón Flecha(1999) precisa:

Mientras que para la concepción constructivista la realidad es un construcción social que depende de los significados que dan las personas, para la opción dialógica la realidad es una construcción humana cuyos significados dependen de las interacciones humanas. Por lo tanto, si para la primera la formación del profesorado se sustenta en el conocimiento del proceso de aprendizaje de los actores y de su forma de construir, la segunda lo cimenia en el conocimiento de los procesos de aprendizaje de los individuos y grupos a través de la construcción interactiva de significados. La opción del aprendizaje dialógico implica, además, una orientación interdisciplinar y una real transformación del contexto donde el respeto a las diferencias se incluye como una de las dimensiones de la educación igualitaria. (pág. 7)

Si creemos que la educación debe formar seres pensantes, debemos comenzar por pensar y pensar juntos. Castoriadis tiene razón cuando afirma que "nunca la acción educadora ha tenido más fuerza política que en la sociedad del "no-pensamiento". Es decir, como somos conscientes, podemos desaprender a pensar como nos enseñaron. Obviamente; cuando todo se opone a ello, es una tarea harto ardua y audaz que algunos llegan a pensar que es paralizante, elitista y teórica. Refiriéndose a este temática V.Forrester (1997) reflexiona:

Hay que desbaratar la trampa de separar lo intelectual de lo visceral, el pensamiento de la emoción...Porque no hay nada más movilizador que el pensamiento. Lejos de representar una triste abdicación es la quintaesencia misma de la acción. No existe una actividad más subversiva ni temida. Y también más difamada, lo cual no es casual ni carece de importancia: el pensamiento es político. El solo hecho de pensar es político. De ahí la lucha incidiosa, y por eso más eficaz, y más intensa en nuestra época, contra el pensamiento. Contra la capacidad de pensar. Pensar es vivir y generar vida nueva". (pág. 63).

En el terreno educativo, indudablemente el decidir pensar está indisolublemente ligado al decidir hacer y sentir que presuponen no solamente la construcción de la acción colectiva sino también la recuperación de la ternura y de los procesos de humanización en las más amplias acepciones del término. La educación es una arena de lucha ideológica: el hombre de la respuesta versus el hombre de la pregunta. 
En nuestro siglo y frente a esta sociedad que se niega a reconocer la diversidad y en aras de reconstruir el sentido, pertinencia y pertenencia de la educación podríamos comenzar a esbozar algunos principios orientadores de una nueva docencia desde la pedagogía crítica que recuperaran la idea de la educación como proceso de apropiación de la historia y de la cultura. a saber: educar para la incertidumbre, la complejidad y la imprevisibilidad; educar para gozar de la vida; educar para la significación, la coherencia y la integralidad; educar para la expresión y la comprensión y, finalmente, educar para la convivencia y la tolerancia.

\section{Referencias}

Delval, Juan(2000) Aprender en la vida y en la escuela, Madrid: Morata.

Flecha, Ramón (1999), Aprendizaje dialógico en la era de la información; Gandía: Editorial Movimiento de Renovación Pedagógica.

Forrester,V. (1997), El horror económico, Buenos Aires: Fondo de Cultura Económica.

Galeano, Eduardo (1999) La escuela del crimen, Montevideo: Semanario Brecha.

Larrosa, Jorge (1995) Escuela, poder y subjetivación, Madrid: Editorial de la Piqueta.

Mardones, J.M. (1998), Desafios para recrear la escuela, Madrid: Editorial PPC.

Morin, Edgar (2000), La cabeza bien puesta. Pensar la reforma, reformar el pensamiento, Buenos Aires: Editorial Nueva Visión.

Rogero, Julio (2000); Materiales de trabajo para una conferencia, mimeo,Valencia. 